This item was submitted to Loughborough's Research Repository by the author.

Items in Figshare are protected by copyright, with all rights reserved, unless otherwise indicated.

\title{
Field modulated anomalous Hall conductivity and planar Hall effect in Co3Sn2S2 nanoflakes
}

PLEASE CITE THE PUBLISHED VERSION

https://doi.org/10.1021/acs.nanolett.0c02219

\section{PUBLISHER}

American Chemical Society (ACS)

VERSION

AM (Accepted Manuscript)

\section{PUBLISHER STATEMENT}

This document is the Accepted Manuscript version of a Published Work that appeared in final form in Nano Letters, copyright $\odot$ American Chemical Society after peer review and technical editing by the publisher. To access the final edited and published work see https://doi.org/10.1021/acs.nanolett.0c02219

\section{LICENCE}

CC BY-NC-ND 4.0

\section{REPOSITORY RECORD}

Yang, Shuo-Ying, Jonathan Noky, Jacob Gayles, Fasil Dejene, Yan Sun, Mathias Dörr, Yurii Skourski, et al.. 2020. "Field Modulated Anomalous Hall Conductivity and Planar Hall Effect in Co3sn2s2 Nanoflakes". Loughborough University. https://hdl.handle.net/2134/13048058.v1. 


\title{
Field modulated anomalous Hall conductivity and
}

\section{planar Hall effect in $\mathrm{Co}_{3} \mathrm{Sn}_{2} \mathrm{~S}_{2}$ nanoflakes}

\author{
Shuo-Ying Yang ${ }^{1}$, Jonathan Noky ${ }^{2}$, Jacob Gayles ${ }^{3,2}$, Fasil Kidane Dejene ${ }^{1,4}$, Yan Sun ${ }^{2}$, Mathias Dörr ${ }^{5}$, \\ Yurii Skourski ${ }^{6}$, Enke Liu ${ }^{2,5}$, Mazhar Nawaz Ali ${ }^{1}$, Claudia Felser ${ }^{2}$, and Stuart S. P. Parkin ${ }^{1 \dagger}$ \\ ${ }^{I}$ Max-Planck Institute of Microstructure Physics, 06120 Halle (Saale), Germany \\ ${ }^{2}$ Max Planck Institute for Chemical Physics of Solids, 00187 Dresden, Germany \\ ${ }^{3}$ Department of Physics, University of South Florida, Tampa, Florida 33620, USA \\ ${ }^{4}$ Department of Physics, Loughborough University, Leicestershire LE11 3TU,UK \\ ${ }^{5}$ Institute of Physics, Chinese Academy of Science, Beijing 100190, China \\ ${ }^{6}$ Dresden University of Technology, 01602 Dresden, Germany \\ ${ }^{7}$ Dresden High Magnetic Field Laboratory (HLD-EMFL), Helmholtz-Zentrum Dresden-Rossendorf, 01328 \\ Dresden, Germany
}

(Dated: August 20th, 2020)

Time-reversal-symmetry-breaking Weyl semimetals (WSMs) have attracted great attention recently because of the interplay between intrinsic magnetism and topologically nontrivial electrons [1-6]. Here, we present anomalous Hall effect (AHE) and planar Hall effect (PHE) studies on nanoflakes of $\mathrm{Co}_{3} \mathrm{Sn}_{2} \mathrm{~S}_{2}$, a magnetic WSM hosting a stacked Kagome lattice. The reduced thickness modifies the magnetic properties of the nanoflake, resulting in a 15-time larger coercive field compared with the bulk [3], and correspondingly also modifies the transport properties. A $22 \%$ enhancement of the intrinsic anomalous Hall conductivity (AHC), as compared to bulk material, was observed. A magnetic field modulated AHC, which may be directly related to the changing Weyl point separation with magnetic field, was also found. Furthermore, we showed that the PHE in a hard magnetic WSM is a complex interplay between ferromagnetism, orbital magnetoresistance and the chiral anomaly. Our findings pave the way for a further understanding of exotic transport features in the burgeoning field of magnetic topological phases.

Keywords: magnetic Weyl semimetal, anomalous Hall effect, planar Hall effect, berry curvature 
The theoretical prediction and experimental realization of Weyl semimetals (WSMs) in various forms have been of great interest in condensed matter physics in the recent past [7-9]. WSMs are characterized by Fermi arcs at their surfaces and bulk Weyl fermions stemming from band splitting driven by spin-orbit coupling (SOC). The topological character of Weyl nodes results in a number of fascinating properties including an uncompensated Berry curvature, which corresponds to an intrinsic AHE $[3,4,10,11]$. In addition, when an external electric field and magnetic field are applied parallel to each other, chiral charge pumping occurs between the two Weyl nodes, which have opposite chiralities, and manifests itself as a negative longitudinal magnetoresistance (NLMR) [12-18], and a planar Hall effect (PHE) [19-29].

Recently, WSMs with intrinsic magnetism have attracted great attention [1-6]. Compared with inversion-symmetry (IS)-breaking WSMs, time-reversal-symmetry (TRS)-breaking WSMs provide a playground where the interplay of intrinsic magnetism, Berry curvature and topological character of Weyl nodes can give rise to rich physical phenomena, such as a Berry curvature induced AHE and anomalous Nernst effect [3, 4], a high-temperature quantum AHE in the 2D limit [30], as well as possible device applications connecting topological physics and thermoelectric devices [31, 32]. The close relationship between TRS-breaking and intrinsic magnetism makes it possible to manipulate the topological order via changes in the magnetic order [11,33-35].

Since the realization of WSMs, most studies have centered around IS-breaking WSMs [36-42]. The transport characteristics of TRS-breaking WSMs (like ferromagnetic WSMs), especially those in which the transport properties can be modified by changing magnetic properties have been sparsely addressed. The prediction that the Kagome lattice compound, $\mathrm{Co}_{3} \mathrm{Sn}_{2} \mathrm{~S}_{2}$ is a ferromagnetic TRS-breaking WSM was recently confirmed by direct observation of its Fermi arcs via ARPES and STM measurements $[43,44]$. In this work, we present detailed magneto-transport measurements on $180 \mathrm{~nm}$ thick $\mathrm{Co}_{3} \mathrm{Sn}_{2} \mathrm{~S}_{2}$ nanoflakes and discuss the intrinsic AHE, longitudinal magnetoresistance (LMR) and PHE in the context of ferromagnetic ordering with extremely large out-of-plane magnetic anisotropy (anisotropy field > 5T). In addition to a $22 \%$ enhancement of the intrinsic AHC compared with the bulk $\mathrm{Co}_{3} \mathrm{Sn}_{2} \mathrm{~S}_{2}$ sample, the reduced thickness increases the coercive field by more than 15 times compared with the bulk, revealing an anomaly in the Hall resistivity which corresponds to a field modulated AHC 
that may be directly related to the Weyl node separation. In addition, we discuss the PHE in a $\mathrm{Co}_{3} \mathrm{Sn}_{2} \mathrm{~S}_{2}$ nanoflake, which arises from a complex interaction of ferromagnetism, orbital magnetoresistance and the chiral anomaly, that has not been shown in other materials or previous analyses [45].

$\mathrm{Co}_{3} \mathrm{Sn}_{2} \mathrm{~S}_{2}$ crystallizes in a rhombohedral structure in which a quasi-2D $\mathrm{Co}_{3} \mathrm{Sn}$ layer is sandwiched between sulfur and tin atoms, as is shown in Figure 1a. The magnetism originates from the cobalt atoms that sit on a Kagome lattice in the $a b$ plane. Figure $1 \mathrm{~b}$ shows a typical $180 \mathrm{~nm}$ thick nanoflake device used in this work, in which the dashed lines outline the nanoflake before Ar ion etching. The current is applied along the $a$-axis. The temperature dependence of the longitudinal resistivity of a nanoflake at $\mu_{0} H=0 \mathrm{~T}$ displays a metallic behavior with a residual resistance ratio $\rho(300$ $\mathrm{K}) / \rho(2 \mathrm{~K})$ of 14 . Ferromagnetic ordering appears below the $T_{C}$ of $171 \mathrm{~K}$, as indicated by the kink in Figure 1c. Figure 1d shows the transverse magnetoresistance (TMR) with $\mu_{0} H$ applied perpendicular to the nanoflake at various temperatures. At temperatures above $100 \mathrm{~K}$ and below the $T_{c}$, the TMR is negative, as typically seen in ferromagnets due to magnetic-field induced suppression of thermal spin fluctuations [47, 48]. At $2 \mathrm{~K}$, the TMR reaches $139 \%$ at $14 \mathrm{~T}$, with no sign of saturation. The TMR also shows clear hysteresis with an extremely large perpendicular magnetic coercive field of $\sim 5 \mathrm{~T}$ at $2 \mathrm{~K}$, which is 15-times larger than the coercive field measured in bulk $\mathrm{Co}_{3} \mathrm{Sn}_{2} \mathrm{~S}_{2}(0.33 \mathrm{~T}$ at $2 \mathrm{~K})$ [3]. We emphasize that the increase in the coercive field is not a result of differences in RRR between the samples. The RRR of the nanoflake of this study is in between the RRRs of the two bulk crystals reported in previous literature $[3,45]$, but the $H_{c}$ of the nanoflake is increased by an order of magnitude compared with the bulk samples (also see supporting information 7). Further magnetization measurements (Supporting Information) reveal that when the field is applied along the in-plane $a$-axis, the in-plane saturation field is as high as $24 \mathrm{~T}$, implying it is extremely hard to align the moments in-plane [49]. This reflects a very large coercive field as well as a large in-plane saturation field which are key to understanding the transport properties of the nanoflakes, as discussed below.

Figure 1e and 1f show a magnified plot of the TMR as indicated by the black dotted square in Figure $1 \mathrm{~d}$. At temperatures higher than $30 \mathrm{~K}$, the TMR increases when $\mu_{0} H$ and effective out-of-plane magnetic moments $M_{z}$ are antiparallel aligned, so that the TMR values drop at the coercive field. Interestingly, at temperatures below $20 \mathrm{~K}$ (see Figure 1f) the TMR decreases when $\mu_{0} H$ and $M_{z}$ are 
antiparallel aligned compared with when they are parallel aligned, therefore it jumps up at the coercive field. Such TMR behavior at $2 \mathrm{~K}$ is not typical for conventional ferromagnets and deserves some special attention. In a semimetal with strong out-of-plane ferromagnetic moments, there are three main contributions to TMR: the first one is the ordinary magnetoresistance (OMR) that is related to the carrier mobilities and scales with $\mu B^{2}$. The second one is a spin-dependent magnetoresistance that results from spin orientation-dependent electron scattering: the overall resistance is relatively high when $\mu_{0} H$ and $M_{z}$ are antiparallel aligned because electron scattering is stronger in this configuration and weaker when $\mu_{0} H$ and $M_{z}$ are aligned. The third contribution originates from the strong out-of-plane moments which act as a fictitious magnetic field, in addition to the external applied magnetic field, and generates an extra OMR. When $\mu_{0} H$ and $M_{z}$ are antiparallel aligned, the effective out-of-plane moment $M_{z}$ results in a reduced effective field and a smaller positive contribution to the OMR. The fact that the TMR at low and high temperatures show different hysteresis behaviors is an indication of the competition between the last two mechanisms at different temperatures. At higher temperatures, scattering mechanisms dominate so that the TMR increases when $\mu_{0} H$ and $M_{z}$ are antiparallel. At low temperatures, contributions from scattering are smaller, making the effective OMR the dominant contribution.

The semi-metallicity of the nanoflake devices is confirmed by the Hall effect measurements (Figure 2 as well as Supporting Information). Figure 2a shows the Hall resistivity at various temperatures below $40 \mathrm{~K}$ where the green line is the overall Hall response $\left(\rho_{x y}\right)$, the dark blue line is the extracted two-carrier ordinary Hall $\left(\rho_{x y}^{o}\right)$ and the red line is the difference between the two $\left(\rho_{x y}^{*}=\right.$ $\left.\rho_{x y}-\rho_{x y}^{o}\right)$. At low temperatures, $\rho_{x y}$ displays a nonlinear behavior on top of a square-shaped hysteresis loop, indicating the co-existence of electron and hole carriers and a single hard magnetic phase, respectively. With increasing temperature, the carrier type gradually becomes hole-dominated (see Supporting Information).

Upon subtracting $\rho_{x y}^{o}, \rho_{x y}^{*}$ smoothly increases when $\mu_{0} H$ and $M_{z}$ are aligned antiparallel and shows a sharp antisymmetric peak at the coercive field. The Hall resistivity anomaly, highlighted by the yellow shading in Figure 2a, decreases with increasing temperature and disappears at about $50 \mathrm{~K}$. This behavior cannot be explained by a typical ferromagnetic AHE, which is linearly dependent on 
magnetization $\left(\rho_{x y}^{A}=R_{S} 4 \pi M_{z}\right)$ : the $M_{z}$ when $\mu_{0} H$ and $M_{z}$ are antiparallel will not exceed the maximum $M_{z}$ achieved when the moments are fully saturated by the external magnetic field, meaning $\rho_{x y}^{A}$ will not be maximized during the antiparallel alignment. The Hall resistivity anomaly resembles the topological Hall effect which has been seen in noncollinear magnetic systems due to a real space Berry phase $[50,51]$ arising from a spatially varying magnetic potential. However, given that numerous studies have shown the robust out-of-plane ferromagnetic ground state in $\mathrm{Co}_{3} \mathrm{Sn}_{2} \mathrm{~S}_{2}$ at low temperatures $[3,4$, $33,52]$, this is unlikely to be the origin of the Hall anomaly.

Another possible reason for the Hall anomaly is a field-dependent AHE arising from a fieldmodulated splitting of Weyl points. To analyze this possibility, it is helpful to convert the Hall resistivity to Hall conductivity which is directly proportional to the magnitude of the uncompensated Berry curvature within the Brillouin zone. Figure $2 \mathrm{~b}$ shows the overall Hall conductivity $\left(\sigma_{x y}=-\frac{\rho_{x y}}{\rho_{x x}^{2}+\rho_{x y}^{2}}\right)$, ordinary Hall conductivity $\left(\sigma_{x y}^{o}\right)$, and the difference between the two $\left(\sigma_{x y}^{*}=\sigma_{x y}-\sigma_{x y}^{o}\right)$ (green, dark blue, and red lines, respectively). In the anti-parallel orientation, $\sigma_{x y}^{*}$ continues to decrease when approaching the coercive field. A decreasing AHC could appear as a natural consequence of Weyl points moving towards each other in a magnetic WSM [7, 10, 11]. If the ferromagnet undergoes a canting of the magnetic moments away from the easy axis resulting in a decreasing out-of-plane magnetization $M_{z}$, the reduction of spin splitting decreases the relative distance between the two Weyl points in $k$ space. As each plane between the two Weyl nodes can be seen as a 2D Chern insulator that has a chiral edge mode contributing a Hall conductance of $\frac{e^{2}}{h}$, the intrinsic AHC becomes smaller when the Weyl points are closer together in $k$-space $\left(\sigma_{x y}^{A}=\frac{K e^{2}}{\pi}\right.$ where $K$ is the distance between two Weyl points) [11]. Given the previously observed robust ferromagnetic ground state at low temperatures in $\mathrm{Co}_{3} \mathrm{Sn}_{2} \mathrm{~S}_{2}[3,4$, 33, 53], we speculate that the field-modulated AHC due to changing Weyl point separation is likely to be the origin of the observed Hall anomaly, and is easily resolved in the nanoflakes because of the increased coercive field compared with the bulk. Future studies on these nanoflakes such as neutron scattering, Lorentz transmission electron microscopy, or magnetic force microscopy to probe the magnetic sublattice and magnetization below $T_{c}$ will help confirm the origin of the anomaly. 
From the Hall measurements, the AHC $\left(\sigma_{x y}^{A}\right)$ at zero field is extracted at each measurement temperature (Supporting Information). Figure 2c shows the dependence of $\sigma_{x y}^{A}$ on the longitudinal conductivity $\sigma_{x x}$. At temperatures below $60 \mathrm{~K}, \sigma_{x y}^{A}$ appears mostly independent of temperature and $\sigma_{x x}$, a hallmark of an intrinsic AHE due to an uncompensated Berry curvature [54, 55]. Surprisingly, the magnitude of $\sigma_{x y}^{A}$ of the nanoflakes reaches as high as $1379 \Omega^{-1} \mathrm{~cm}^{-1}$ (See Supporting Information), approximately $22 \%$ higher than the $\sigma_{x y}^{A}$ of bulk $\mathrm{Co}_{3} \mathrm{Sn}_{2} \mathrm{~S}_{2}$ [3]. Previous DFT calculations show that the magnitude of intrinsic AHC is highly sensitive to the local magnetic moment of Co: $\sigma_{x y}^{A}(0.33$ $\mu \mathrm{bohr} / \mathrm{Co})=1310 \mathrm{ohm}^{-1} \mathrm{~cm}^{-1}[4]$ while $\left.\sigma_{x y}^{A}(0.30 \mu \mathrm{bohr} / \mathrm{Co})=1110 \mathrm{ohm}^{-1} \mathrm{~cm}^{-1}[3]\right)$. The origin of the AHC enhancement may be linked to a larger local moment in these nanoflakes merits future thicknessdependent studies of the AHE and coercive field.

Having established that $\mathrm{Co}_{3} \mathrm{Sn}_{2} \mathrm{~S}_{2}$ nanoflakes display an intrinsic AHE corresponding to the magnetically driven Weyl states, we now discuss the chiral anomaly in the nanoflakes through the PHE and LMR measurements, which have been predicted to reveal chiral charge transport in WSMs [12, 13, $19,20]$ providing inherent current inhomogeneity is excluded. Figure $3 \mathrm{c}$ inset shows a typical schematic for the PHE measurements: $\mu_{0} H$ rotates in the $a b$ plane while the longitudinal and transverse voltages are measured simultaneously, which corresponds to the anisotropic magnetoresistance (AMR) and the change in planar Hall resistivity, respectively. After processing the data to get rid of the effect of misalignments (Supporting Information), the angular dependence of the AMR and PHE within the temperature and field ranges of this study can be well-described by:

$$
\begin{aligned}
\rho_{x x}^{A M R} & =\rho_{\perp}-\Delta \rho_{x y} \cos ^{2} \varphi \\
A M R_{\text {ratio }} & =\frac{\rho_{x x, \varphi}^{A M R}-\rho_{x x, \perp}^{A M R}}{\rho_{x x, \perp}^{A M R}} \times 100 \% \\
\rho_{x y}^{P H E} & =-\Delta \rho_{x y} \sin \varphi \cos \varphi
\end{aligned}
$$

where $\rho_{\perp}$ and $\rho_{\|}$are the longitudinal resistivity when $\mu_{0} H$ is perpendicular or parallel to $E$, respectively, and $\Delta \rho_{x y}=\rho_{\perp}-\rho_{\|}$describes the resistivity anisotropy. Figure 3a and 3b show the angular dependence of the AMR ratio and the planar Hall resistivity measured at $2 \mathrm{~K}$ for $\mu_{0} H$ varying from $1 \mathrm{~T}$ to $9 \mathrm{~T}$. The black dotted lines show fits of the AMR ratio and the PHE with equations (1) and (2). At $2 \mathrm{~K}$, the extracted $\Delta \rho_{x y}$ increases with increasing field, exhibiting a power-law dependence with 
an exponent of 1.81. Figure $3 \mathrm{~d}$ and $3 \mathrm{e}$ show the angular dependence of AMR and planar Hall resistivity at $9 \mathrm{~T}$ in temperatures from $2 \mathrm{~K}$ to $100 \mathrm{~K}$ fitted with Eq. (2). The extracted $\Delta \rho_{x y}$ increases with decreasing temperature and is observable at the highest temperature of $100 \mathrm{~K}$.

In a ferromagnetic WSM, there are three likely microscopic mechanisms to account for the observed PHE: a) spin-dependent scattering in ferromagnets, which stems from the rotation of the magnetization with respect to the current direction, that changes the band occupation and thus scattering rates [56-59], b) anisotropic OMR, which arises from the anisotropy in Fermi pockets leading to $\rho_{\perp}>$ $\rho_{\|}>0[18,28,29]$ and c) the chiral anomaly effect, where chiral charge pumping results in an enhanced conductivity for certain alignments of the magnetic and electric fields giving riseto a PHE [19, 20]. Importantly, the band structures calculated along high symmetry lines including SOC with magnetization $(M)$ aligned along [100], [010] and [001] directions (Figure 4a) show that $\mathrm{Co}_{3} \mathrm{Sn}_{2} \mathrm{~S}_{2}$ is a WSM regardless of its $M$ direction within the ferromagnetic state (i.e. $M\|110, M\| 010$ or $M \| 001$ ). Thus $\mathrm{Co}_{3} \mathrm{Sn}_{2} \mathrm{~S}_{2}$ is unlike a typical nonmagnetic WSM which has anisotropic OMR and chiral anomaly contributions (i.e. $\mathrm{Cd}_{3} \mathrm{As}_{2}$ or $\mathrm{WTe}_{2}[21,25]$ ), or a trivial semimetal which only has orbital contribution (i.e. Bi [18]). Only magnetic WSMs have all three possible origins, and their complex interactions and contributions to the PHE are now examined in detail.

The PHE in ferromagnets depends on the current direction relative to the in-plane magnetization and can be described by [59]:

$$
\rho_{x y}^{F M}=k\left(M_{\|}\right)^{2} \sin \varphi^{*} \cos \varphi^{*}=k(M \sin \alpha)^{2} \sin \varphi^{*} \cos \varphi^{*}
$$

in which $k$ is a constant related to AMR arising from the ferromagnetism, $M_{\|}$is the in-plane projection of the magnetization $M, \alpha$ is the angle between $M$ and the out-of-plane axis and $\varphi^{*}$ is the angle between the applied electric field $E$ and $M_{\|}$(Supporting information). Since $\mathrm{Co}_{3} \mathrm{Sn}_{2} \mathrm{~S}_{2}$ is a ferromagnet below $171 \mathrm{~K}$, it could well be possible that ferromagnetism is the main origin of the observed PHE. However, we argue that the contribution from ferromagnetism is small, for the following reasons. First of all, the magnitude of PHE is directly related to $M_{\|}$. The $M$ of $\mathrm{Co}_{3} \mathrm{Sn}_{2} \mathrm{~S}_{2}$ is $65.2 \mathrm{emu} / \mathrm{cm}^{3}$ and the in-plane saturation magnetic field $\mu_{0} H_{c}$ is larger than $24 \mathrm{~T}$ (see Supporting Information). This is a significantly lower $M$ and higher $\mu_{0} H_{c}$ than conventional ferromagnetic systems in which a PHE has been observed (i.e. $M\left(\right.$ Py) $\approx 750 \mathrm{emu} / \mathrm{cm}^{3} ; \mu_{0} H_{c}$ (Py) $\approx 10$ Oe). From Eq.(3), assuming a uniform canting of the 
moment, $M_{\|}=36.2 \mathrm{emu} / \mathrm{cm}^{3}$ at $2 \mathrm{~K}$ and $9 \mathrm{~T}$, making the magnitude of $\Delta \rho_{x y}$ at least an order of magnitude smaller than a conventional ferromagnet based PHE. In fact, right below $T_{c}$ where the magnetic moments preferentially align more in the plane of the flake than at lower temperatures, no PHE was observed. Secondly, the AMR ratio in $\mathrm{Co}_{3} \mathrm{Sn}_{2} \mathrm{~S}_{2}$ is relatively large, i.e. $-35 \%$, an order of magnitude larger than those in most conventional ferromagnetic metals and half-metallic ferromagnets, for which the AMR is typically only a few percent (e.g. $\mathrm{Fe}=0.3 \% ; \mathrm{Ni}=2.2 \% ; \mathrm{La}_{0.7} \mathrm{Sr}_{0.3} \mathrm{MnO}_{3}=-0.15 \%$ [60]). Thirdly, in many conventional ferromagnets, $\rho_{\perp}$ is smaller than $\rho_{\|}$when the dominant $s-d$ scattering process is spin-flip scattering $[48,60,61]$, whereas we find that $\rho_{\perp}$ is larger than $\rho_{\|}$for $\mathrm{Co}_{3} \mathrm{Sn}_{2} \mathrm{~S}_{2}$. Lastly, if PHE of such a shape is due to ferromagnetism, it should track in temperature with magnetization and be observable immediately below the $T_{c}$. We observe that the PHE in $\mathrm{Co}_{3} \mathrm{Sn}_{2} \mathrm{~S}_{2}$ appears below $100 \mathrm{~K}$, which is far below the ferromagnetic ordering temperature. Thus, we conclude that ferromagnetism is not the main contribution of the PHE in $\mathrm{Co}_{3} \mathrm{Sn}_{2} \mathrm{~S}_{2}$.

However, the strong out-of-plane anisotropy makes it hard to fully disentangle the other two contributions. Figure $4 \mathrm{~b}$ and $4 \mathrm{c}$ show the LMR and TMR with $\mu_{0} H$ applied in-plane, parallel and perpendicular to $E$ at various temperatures. Below $50 \mathrm{~K}$, the LMR has a net weakly positive field dependence while above $50 \mathrm{~K}$ an NLMR can be seen. At $100 \mathrm{~K}$, both LMR and TMR are negative and show hysteresis. In the case of a non-magnetic isotropic IS-breaking WSM where only the chiral anomaly is present, $\rho_{\|}$is negative due to the chiral-anomaly-induced NLMR, giving rise to a finite PHE. Previous studies have shown that this NLMR can be suppressed by a small angle between $E$ and $\mu_{0} H$ $[16,62]$. In a TRS-breaking magnetic WSM with moments pointing strongly out-of-plane (i.e. a hard magnet like $\mathrm{Co}_{3} \mathrm{Sn}_{2} \mathrm{~S}_{2}$ ), applying an in-plane field only cants the moments, leaving large out-of-plane components, shown by the red arrow in the inset to Figure $4 \mathrm{~b}$. These out-of-plane moments can act as a local out-of-plane field and generate a Lorentz force, thereby giving an additional positive contribution to $\rho_{\|}$, resulting in a net $\rho_{\|}$with a weakly positive dependence on field, making the low-temperature NLMR absent in the $\mathrm{Co}_{3} \mathrm{Sn}_{2} \mathrm{~S}_{2}$ nanoflakes of this study. At higher temperatures, it is easier to align the moments in-plane, thus the local out-of-plane field created by the $M_{z}$ can vanish, making the chiral anomaly driven NLMR possible to observe. However, magnetic field induced suppression of thermal spin fluctuations may also give rise to a NLMR at high temperatures, making the chiral anomaly and 
the ferromagnetic component of the NLMR hard to disentangle. To be able to observe the chiral anomaly through PHE or LMR measurements in WSMs, the OMR needs to be small [23]. In previously measured bulk crystal PHE studies, the NLMR was not observed (likely due to a large OMR), but the OMR contribution to the PHE was ignored [45]. For antiferromagnetic WSMs, it may be possible to probe the chiral anomaly driven PHE below the spin flop transition. For ferromagnetic WSMs, one needs to fully saturate the ferromagnetism and get rid of any out-of-plane moment to be able to unambiguously probe the chiral anomaly driven PHE.

In summary, we have carried out detailed magneto-transport measurements in $\mathrm{Co}_{3} \mathrm{Sn}_{2} \mathrm{~S}_{2}$ nanoflakes to elucidate the origins of AHE and PHE in a magnetic WSM with strong out-of-plane anisotropy as large as $5 \mathrm{~T}$. The reduced thickness increases the coercive field of the nanoflake by 15time, affecting the transport properties: we observed a large intrinsic AHC that is $22 \%$ larger than bulk samples, and a field-dependent Hall anomaly, the magnitude of which cannot be explained by classical magnetization strength driven AHE. It can, however, be related to the extent of the Weyl point separation in $k$-space, a direct manifestation of Weyl physics. Finally, we showed that the PHE and LMR in the $\mathrm{Co}_{3} \mathrm{Sn}_{2} \mathrm{~S}_{2}$ nanoflake are a result of a complex interplay between contributions from ferromagnetism, orbital magnetoresistance and the chiral anomaly. Our findings provide a direction for future understanding of exotic topological transport in $\mathrm{Co}_{3} \mathrm{Sn}_{2} \mathrm{~S}_{2}$ as well as other TRS-breaking WSMs. 
Material growth and device fabrication. $\mathrm{Co}_{3} \mathrm{Sn}_{2} \mathrm{~S}_{2}$ nanoflakes were grown using a chemical-vaportransport (CVT) method on $\mathrm{Al}_{2} \mathrm{O}_{3}$ (0001) substrates. Surface morphology and thickness of selected flakes were measured using a Bruker Multimode 8 atomic force microscopy (AFM). The flake used in this work was $180 \mathrm{~nm}$ thick (Supporting Information). Selected flakes were fabricated into Hall bar geometry devices via standard e-beam lithography and Ar ion etching. Afterwards, Ru/Au $(10 \mathrm{~nm} / 200$ nm) electrodes were sputter deposited as contacts.

Magneto-resistivity measurements. The magneto-resistivity measurements were performed in a PPMS DynaCool (Quantum Design) with a magnetic field up to $14 \mathrm{~T}$ using the 'DC Resistivity' option. The rotator insert (Quantum Design) was used to tilt the angle between the magnetic field and the current.

Density functional theory (DFT) calculations. The ab-initio DFT calculations were performed using the package VASP. 
a

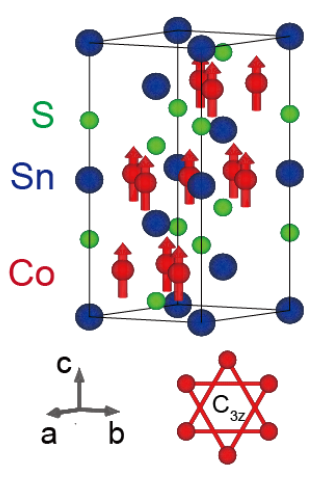

d

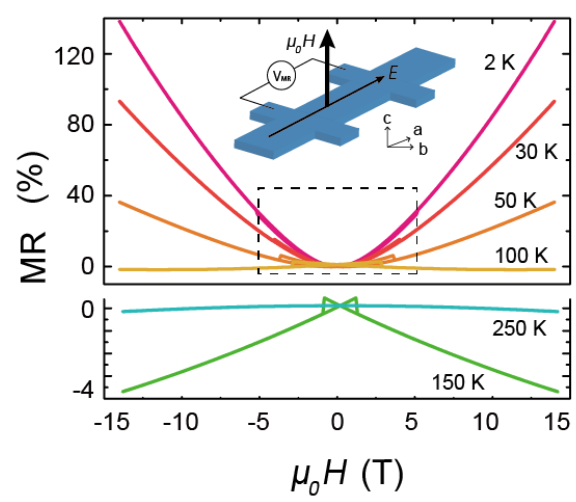

b
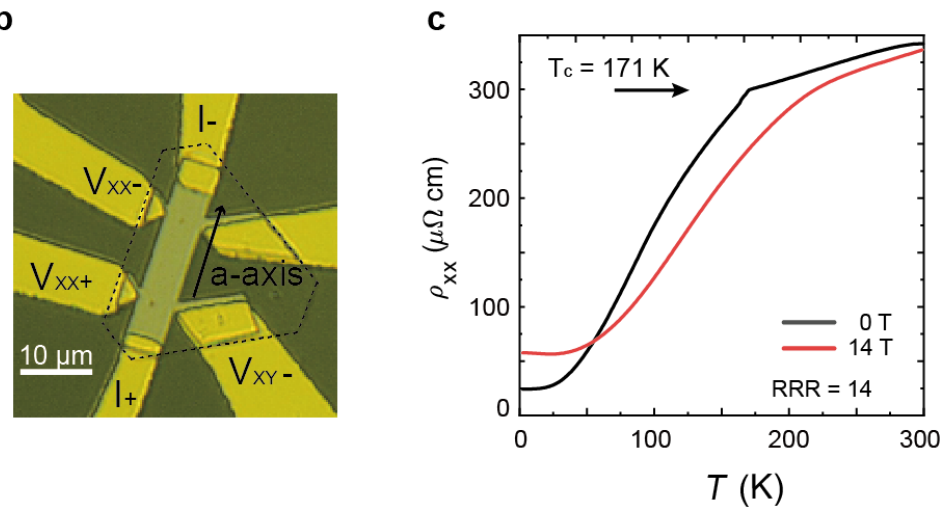

e

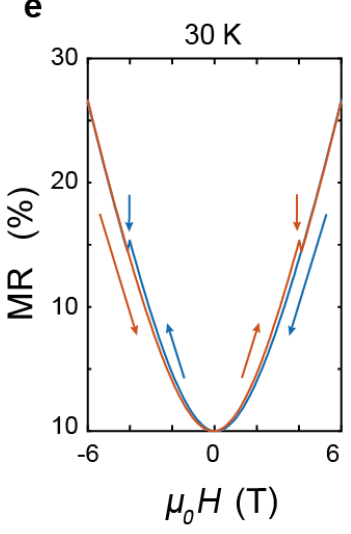

f

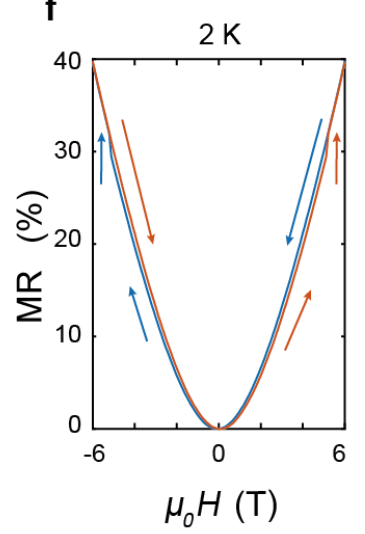

Figure 1. Basic transport characterization of a $\mathrm{Co}_{3} \mathrm{Sn}_{2} \mathrm{~S}_{2}$ nanoflake. (a) The crystal structure of $\mathrm{Co}_{3} \mathrm{Sn}_{2} \mathrm{~S}_{2}$, in which an out-of-plane ferromagnetic Kagome lattice is formed with cobalt atoms. (b) An optical image of a typical device used in this work. The black dotted line shows the contour of the nanoflake before Ar ion etching. (c) The temperature dependence of longitudinal resistivity at a magnetic field of $0 \mathrm{~T}$ and $14 \mathrm{~T}$. The kink at $171 \mathrm{~K}$ corresponds to the $T_{c}$. (d) TMR measured at various temperatures with field applied out-of-plane perpendicular to the nanoflake, which changes from positive to negative with increasing temperature. (e, f) Zoom in plot of the TMR at $30 \mathrm{~K}$ and $2 \mathrm{~K}$ highlighted in the black box in (d). The blue curves are the TMR measured when the field is swept from positive to negative and the orange curves are the TMR when the field is swept from negative to positive. 

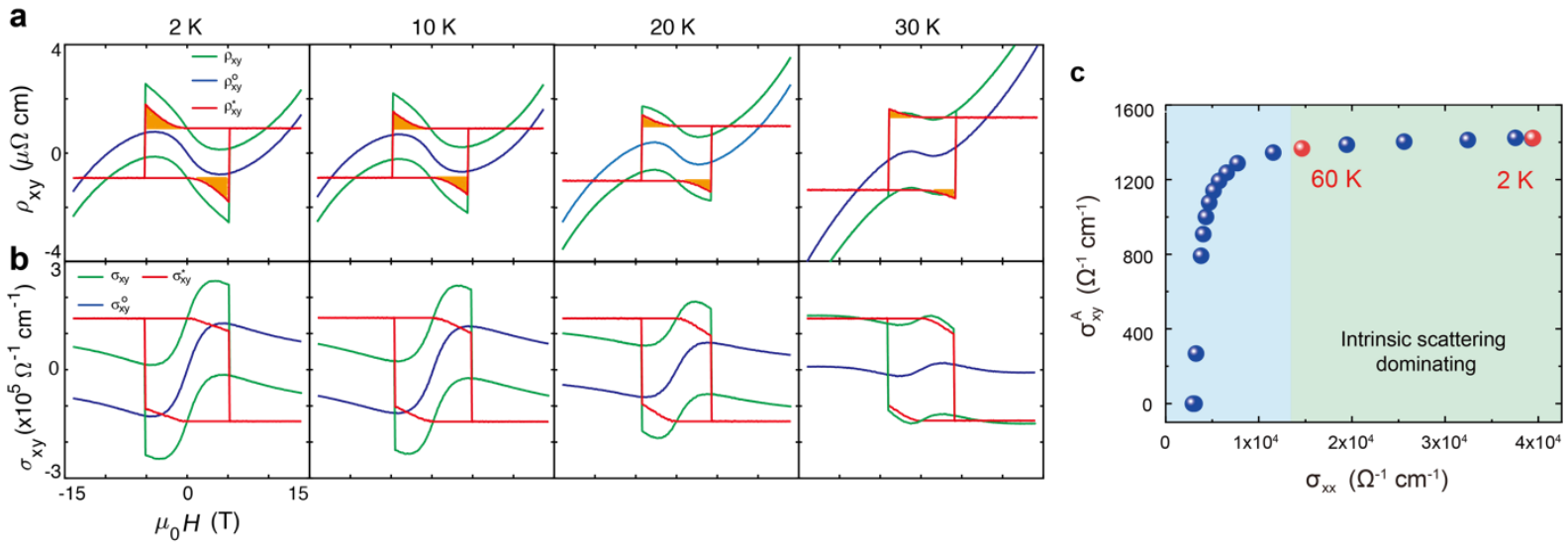

Figure 2. Field dependence of Hall resistivity and Hall conductivity measured at various temperatures.

(a) The overall Hall resistivity $\rho_{x y}$ (green line), two-carrier ordinary Hall resistivity $\rho_{x y}^{o}$ (dark blue line) and the difference between the two $\rho_{x y}^{*}=\rho_{x y}-\rho_{x y}^{o}$ (red). The yellow shadow shows the additional Hall resistivity component, which decreases with increasing temperature. (b) The overall Hall conductivity $\sigma_{x y}$ (green line), two-carrier ordinary Hall conductivity $\sigma_{x y}^{o}$ (dark blue) and the difference between the two (red). When the external field is antiparallel to $M_{z}$, the magnitude of the $\sigma_{x y}^{*}$ decreases with increasing field. (c) $\sigma_{x x}$ dependence of $\sigma_{x y}^{A} . \sigma_{x y}^{A}$ is mostly independent of longitudinal $\sigma_{x x}$ below $60 \mathrm{~K}$, indicating its intrinsic origin. 


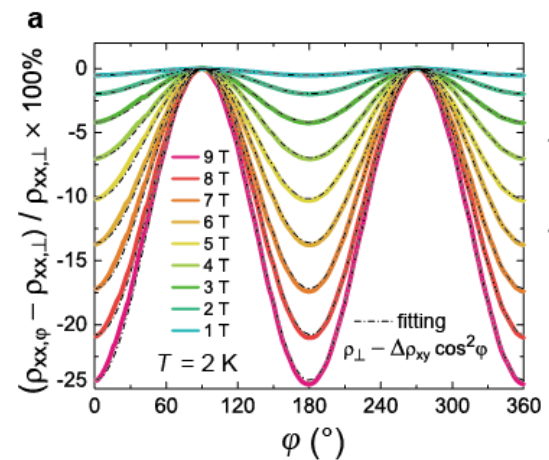

d

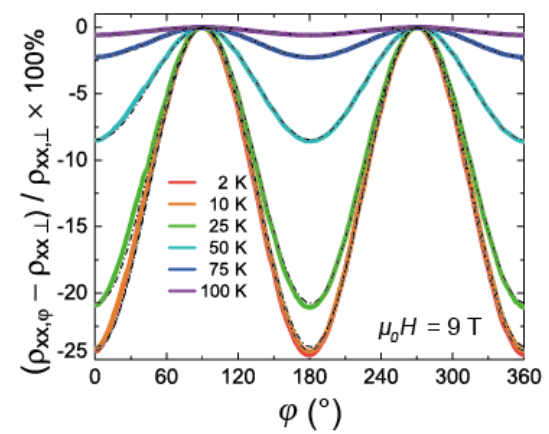

b

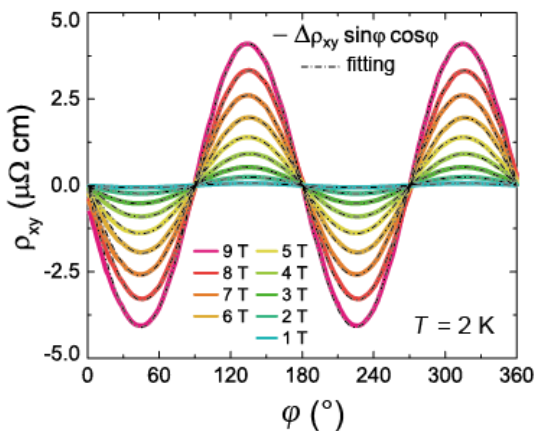

e

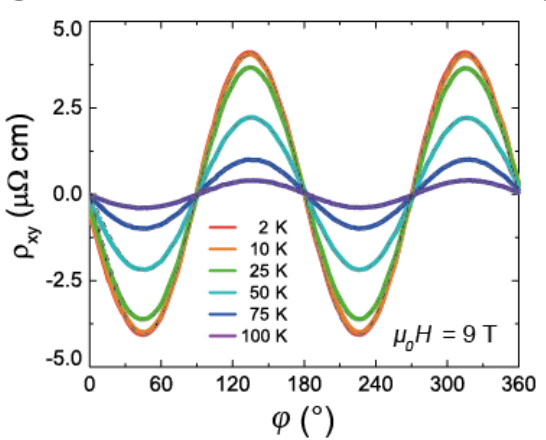

C

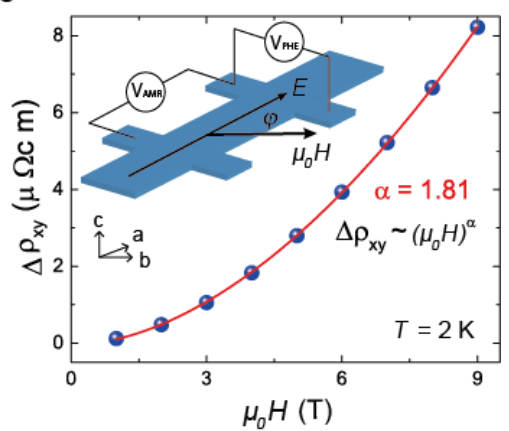

f

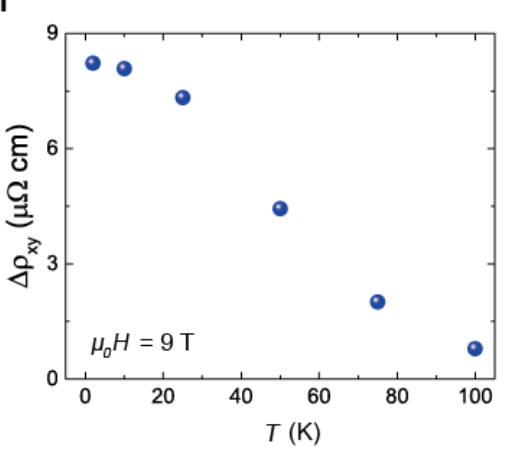

Figure 3: Anisotropic magnetoresistance and planar Hall effect. (a, b) AMR ratio and intrinsic planar Hall resistivity measured at $2 \mathrm{~K}$ in magnetic fields from $1 \mathrm{~T}$ through $9 \mathrm{~T}$. The black dotted lines show the fitting of the data with equations shown in the plot. (c) Field dependence of $\Delta \rho_{x y}$ obtained from fitting, which follows a power law with an exponent of 1.81. The inset shows schematics of the Hall bar device and the measurement configuration. The sample is rotated in the $a b$-plane while $\mathrm{V}_{\mathrm{xx}}$ and $\mathrm{V}_{\mathrm{PHE}}$ are measured simultaneously. (d, e) AMR ratio and planar Hall resistivity measured at $9 \mathrm{~T}$ under temperatures ranging from $2 \mathrm{~K}$ to $100 \mathrm{~K}$. The black dotted lines show the fitting with equations shown in the plot. (f) Temperature dependence of $\Delta \rho_{x y}$ obtained from fitting in Fig. 2e. 

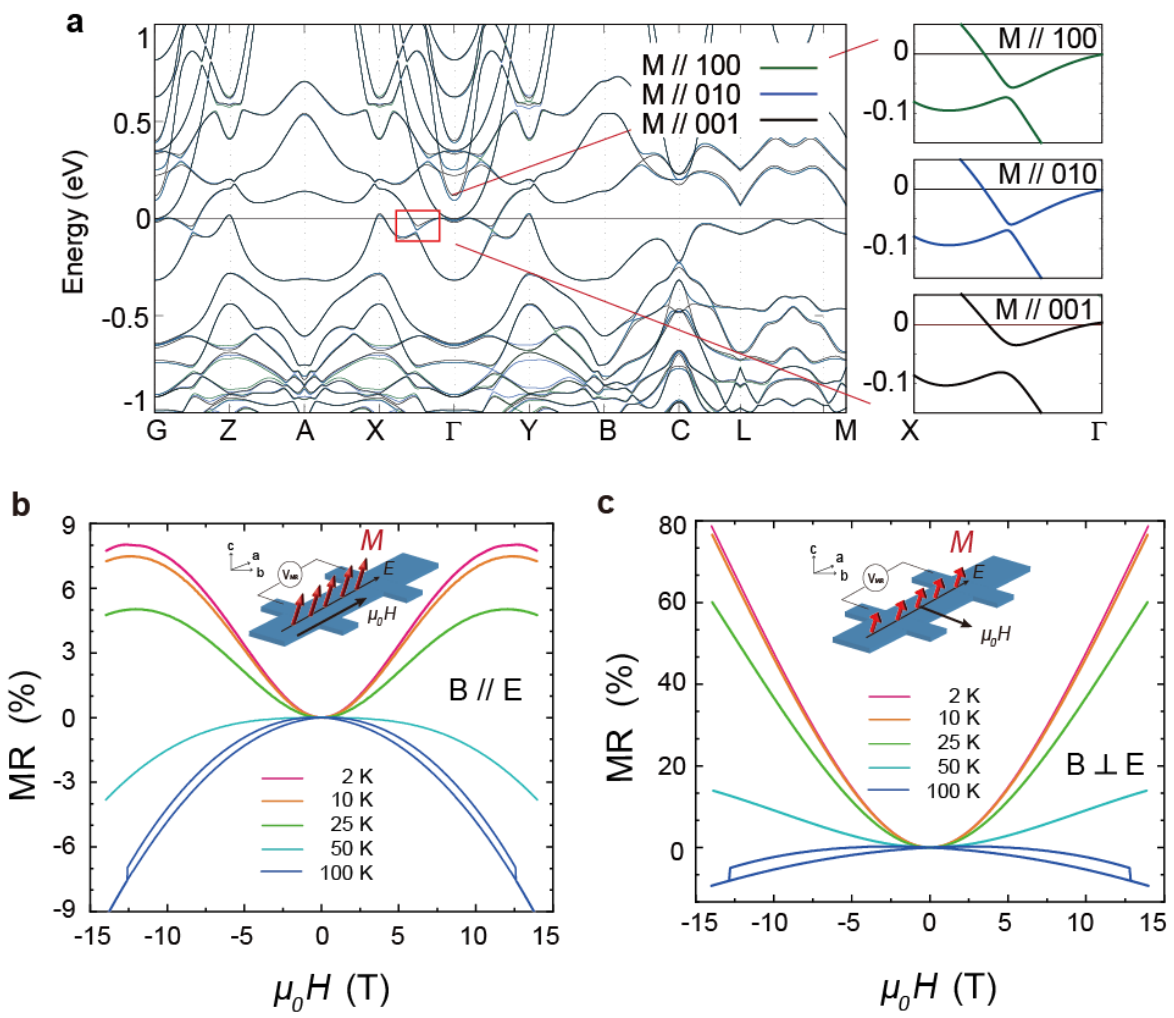

Figure 4. Band structure calculation, magnetoresistance with field applied in-plane parallel or perpendicular to the current direction at different temperatures. (a) Band structure calculation along high symmetry lines of $\mathrm{Co}_{3} \mathrm{Sn}_{2} \mathrm{~S}_{2}$ with $M$ aligned along [100], [010] and [001] directions including spin-orbit coupling. The red box highlights the gapped nodal line resulting in Weyl crossings which appear off high symmetry axes in all three cases. (b) Longitudinal magnetoresistance measured with $\mu_{0} H$ parallel to $E$ at various temperatures. (c) Transverse magnetoresistance measured with $\mu_{0} H$ applied in-plane and perpendicular to $E$ at various temperatures. 


\section{Acknowledgments}

We thank Yuanfeng Xu and Kai Chang for useful discussions. This work was funded by the Deutsche Forschungsgemeinschaft SPP 1666. M.N.A acknowledges Alexander von Humboldt Foundation Sofia Kovalevskaja Award, the MINERVA ARCHES Award. E.L. thanks the National Natural Science Foundation of China (No. 11974394). We acknowledge HLD at HZDR, a member of the European Magnetic Field Laboratory (EMFL) for the high magnetic field measurements.

\section{Author contributions}

S.Y.Y., E.L. C.F. and S.S.P.P. conceived the experiments. E.L. grew the nanoflake crystals. S.Y.Y. fabricated the device and performed the transport measurements. J.N., J.G. and Y.S. performed the DFT calculation. S.Y.Y., F.K.D., M.N.A analyzed the data. S.Y.Y., F.K.D., M.N.A and S.S.P.P. wrote the manuscript. M.N.A. C.F. and S.S.P.P. supervised the project.

\section{References:}

1. Hirschberger, M., et al., The chiral anomaly and thermopower of Weyl fermions in the half-Heusler GdPtBi. Nat Mater, 2016. 15(11): p. 1161-1165.

2. Kuroda, K., et al., Evidence for magnetic Weyl fermions in a correlated metal. Nat Mater, 2017. 16(11): p. 1090-1095.

3. Liu, E., et al., Giant anomalous Hall effect in a ferromagnetic Kagome-lattice semimetal. Nat Phys, 2018. 14(11): p. 1125-1131.

4. Wang, Q., et al., Large intrinsic anomalous Hall effect in half-metallic ferromagnet Co3Sn2S2 with magnetic Weyl fermions. Nat Commun, 2018. 9(1): p. 3681.

5. Sakai, A., et al., Giant anomalous Nernst effect and quantum-critical scaling in a ferromagnetic semimetal. Nature Physics, 2018. 14(11): p. 1119-+.

6. Ye, L.D., et al., Massive Dirac fermions in a ferromagnetic kagome metal. Nature, 2018. 555(7698): p. 638-+.

7. Armitage, N.P., E.J. Mele, and A. Vishwanath, Weyl and Dirac semimetals in threedimensional solids. Reviews of Modern Physics, 2018. 90(1).

8. Yan, B. and C. Felser, Topological materials: Weyl semimetals. Annual Review of Condensed Matter Physics, 2017. 8: p. 337-354.

9. Hasan, M.Z., et al., Discovery of Weyl fermion semimetals and topological Fermi arc states. Annual Review of Condensed Matter Physics, 2017. 8: p. 289-309.

10. Burkov, A.A., Anomalous Hall effect in Weyl metals. Phys Rev Lett, 2014. 113(18): p. 187202.

11. Yang, K.Y., Y.M. Lu, and Y. Ran, Quantum Hall effects in a Weyl semimetal: Possible application in pyrochlore iridates. Physical Review B, 2011. 84(7).

12. Burkov, A., Negative longitudinal magnetoresistance in Dirac and Weyl metals. Physical Review B, 2015. 91(24): p. 245157.

13. Son, D. and B. Spivak, Chiral anomaly and classical negative magnetoresistance of Weyl metals. Physical Review B, 2013. 88(10): p. 104412.

14. Xiong, J., et al., Evidence for the chiral anomaly in the Dirac semimetal Na3Bi. Science, 2015. 350(6259): p. 413-416. 
15. Huang, X.C., et al., Observation of the Chiral-Anomaly-Induced Negative Magnetoresistance in 3D Weyl Semimetal TaAs. Physical Review X, 2015. 5(3).

16. Wang, Y., et al., Gate-tunable negative longitudinal magnetoresistance in the predicted type-II Weyl semimetal WTe2. Nat Commun, 2016. 7: p. 13142.

17. Zhang, C.L., et al., Signatures of the Adler-Bell-Jackiw chiral anomaly in a Weyl fermion semimetal. Nature Communications, 2016. 7.

18. Liang, S.H., et al., Experimental Tests of the Chiral Anomaly Magnetoresistance in the Dirac-Weyl Semimetals Na3Bi and GdPtBi. Physical Review X, 2018. 8(3).

19. Nandy, S., et al., Chiral Anomaly as the Origin of the Planar Hall Effect in Weyl Semimetals. Physical Review Letters, 2017. 119(17).

20. Burkov, A.A., Giant planar Hall effect in topological metals. Physical Review B, 2017. 96(4).

21. Li, H., et al., Giant anisotropic magnetoresistance and planar Hall effect in the Dirac semimetal Cd3As2. Physical Review B, 2018. 97(20).

22. $\mathrm{Wu}, \mathrm{M}$., et al., Probing the chiral anomaly by planar Hall effect in Dirac semimetal Cd3As2 nanoplates. Physical Review B, 2018. 98(16).

23. Kumar, N., et al., Planar Hall effect in the Weyl semimetal GdPtBi. Physical Review B, 2018. 98(4).

24. Li, P., et al., Giant Planar Hall Effect in the Dirac Semimetal ZrTe5. 2018.

25. Li, P., et al., Anisotropic planar Hall effect in the type-II topological Weyl semimetal WTe2. Physical Review B, 2019. 100(20).

26. Li, Z.G., et al., Planar Hall effect in PtSe2. Journal of Applied Physics, 2020. 127(5).

27. Singha, R., et al., Planar Hall effect in the type-II Dirac semimetal VAl3. Physical Review B, 2018. 98(8).

28. Liang, D.D., et al., Origin of planar Hall effect in type-II Weyl semimetal MoTe2. Aip Advances, 2019. 9(5).

29. Liu, Q.Q., et al., Nontopological origin of the planar Hall effect in the type-II Dirac semimetal NiTe2. Physical Review B, 2019. 99(15).

30. Muechler, L., et al., Emerging chiral edge states from the confinement of a magnetic Weyl semimetal in Co 3 Sn 2 S 2. Physical Review B, 2020. 101(11): p. 115106.

31. Guin, S.N., et al., Zero-Field Nernst Effect in a Ferromagnetic Kagome-Lattice WeylSemimetal Co3Sn2S2. Advanced Materials, 2019. 31(25).

32. Li, G.W., et al., Surface states in bulk single crystal of topological semimetal Co3Sn2S2 toward water oxidation. Science Advances, 2019. 5(8).

33. Guguchia, Z., et al., Tunable anomalous Hall conductivity through volume-wise magnetic competition in a topological kagome magnet. Nat Commun, 2020. 11(1): p. 559.

34. Lachman, E., et al., Exchange biased anomalous Hall effect driven by frustration in a magnetic kagome lattice. Nature communications, 2020. 11(1): p. 1-8.

35. Yang, R., et al., Magnetization-Induced Band Shift in Ferromagnetic Weyl Semimetal Co 3 Sn 2 S 2. Physical Review Letters, 2020. 124(7): p. 077403.

36. Lv, B.Q., et al., Experimental Discovery of Weyl Semimetal TaAs. Physical Review X, 2015. 5(3).

37. $\mathrm{Xu}, \mathrm{S} . \mathrm{Y}$., et al., Discovery of a Weyl fermion semimetal and topological Fermi arcs. Science, 2015. 349(6248): p. 613-617.

38. $\mathrm{Xu}, \mathrm{S} .-\mathrm{Y}$., et al., Experimental discovery of a topological Weyl semimetal state in TaP. Science advances, 2015. 1(10): p. e1501092.

39. Lv, B.Q., et al., Observation of Weyl nodes in TaAs. Nature Physics, 2015. 11(9): p. 724-+.

40. Deng, K., et al., Experimental observation of topological Fermi arcs in type-II Weyl semimetal MoTe 2. Nature Physics, 2016. 12(12): p. 1105-1110. 
41. Huang, L., et al., Spectroscopic evidence for a type II Weyl semimetallic state in MoTe2. Nature Materials, 2016. 15(11): p. 1155-+.

42. Ali, M.N., et al., Large, non-saturating magnetoresistance in WTe. Nature, 2014. 514(7521): p. 205.

43. Liu, D.F., et al., Magnetic Weyl semimetal phase in a Kagome crystal. Science, 2019. 365(6459): p. 1282-+.

44. Morali, N., et al., Fermi-arc diversity on surface terminations of the magnetic Weyl semimetal Co3Sn2S2. Science, 2019. 365(6459): p. 1286-+.

45. Gopal, R. and Y. Singh, Observation of planar Hall effect in the ferromagnetic Weyl semimetal Co3Sn2S2. Journal of Magnetism and Magnetic Materials, 2020. 502: p. 166547.

46. Ding, L.C., et al., Intrinsic Anomalous Nernst Effect Amplified by Disorder in a HalfMetallic Semimetal. Physical Review X, 2019. 9(4).

47. Yamada, H. and S. Takada, Negative Magnetoresistance of Ferromagnetic Metals Due to Spin Fluctuations. Progress of Theoretical Physics, 1972. 48(6a): p. 1828-1848.

48. Smit, J., Magnetoresistance of Ferromagnetic Metals and Alloys at Low Temperatures. Physica, 1951. 17(6): p. 612-627.

49. Shen, J., et al., On the anisotropies of magnetization and electronic transport of magnetic Weyl semimetal Co3Sn2S2. Applied Physics Letters, 2019. 115(21): p. 212403.

50. Neubauer, A., et al., Topological Hall Effect in the A Phase of MnSi. Physical Review Letters, 2009. 102(18).

51. Kanazawa, N., et al., Large Topological Hall Effect in a Short-Period Helimagnet MnGe. Physical Review Letters, 2011. 106(15).

52. Kassem, M.A., et al., Low-field anomalous magnetic phase in the kagome-lattice shandite C o 3 S n 2 S 2. Physical Review B, 2017. 96(1): p. 014429.

53. Mohamed A. Kassem, Y.T., Takeshi Waki, Hiroyuki Nakamura, Low-field anomalous magnetic phase in the kagome-lattice shandite Co3Sn2S2. Physical Review B, 2017. 014429.

54. Miyasato, T., et al., Crossover behavior of the anomalous Hall effect and anomalous nernst effect in itinerant ferromagnets. Phys Rev Lett, 2007. 99(8): p. 086602.

55. Onoda, S., N. Sugimoto, and N. Nagaosa, Intrinsic versus extrinsic anomalous Hall effect in ferromagnets. Phys Rev Lett, 2006. 97(12): p. 126602.

56. K L Yau, J.T.H.C., The planar Hall effect in thin foils of Ni-Fe alloy. J. Phys. F: Met. Phys, 1971. 138.

57. Tang, H.X., et al., Giant planar Hall effect in epitaxial (Ga,Mn)As devices. Physical Review Letters, 2003. 90(10).

58. Seemann, K.M., et al., Origin of the Planar Hall Effect in Nanocrystalline Co60Fe20B20. Physical Review Letters, 2011. 107(8).

59. Nazmul, A.M., et al., Planar Hall effect and uniaxial in-plane magnetic anisotropy in Mn delta-doped GaAs/p-AlGaAs heterostructures. Physical Review B, 2008. 77(15).

60. Kokado, S., et al., Anisotropic Magnetoresistance Effects in Fe, Co, Ni, Fe4N, and HalfMetallic Ferromagnet: A Systematic Analysis. Journal of the Physical Society of Japan, 2012. 81(2).

61. Mcguire, T.R. and R.I. Potter, Anisotropic Magnetoresistance in Ferromagnetic 3d Alloys. Ieee Transactions on Magnetics, 1975. 11(4): p. 1018-1038.

62. dos Reis, R.D., et al., On the search for the chiral anomaly in Weyl semimetals: the negative longitudinal magnetoresistance. New Journal of Physics, 2016. 18. 